\title{
On the performance of non-orthogonal multiple access (NOMA) using FPGA
}

\author{
Mohamad A. Ahmed, Khalid F. Mahmmod, Mohammed M. Azeez
}

College of Electronics Engineering, Ninevah University, P.O. 41002, Mosul, Iraq

\begin{tabular}{l}
\hline Article Info \\
\hline Article history: \\
Received Jul 14, 2019 \\
Revised Oct 24, 2019 \\
Accepted Nov 1, 2019
\end{tabular}

Keywords:

Additive white gaussian noise (AWGN)

Field-programmable gate array (FPGA)

Non-orthogonal multiple access (NOMA)

Successive-interference cancellation (SIC)

\section{Corresponding Author:} \begin{abstract}
In this paper, non-orthogonal multiple access (NOMA) is designed and implemented for the fifth generation (5G) of multi-user wireless communication. Field-programmable gate array (FPGA) is considered for the implementation of this technique for two users. NOMA is applied in downlink phase of the base-station (BS) by applying power allocation mechanism for far and near users, in which one signal contains the superposition of two scaled signals depending on the distance of each user from the BS. We assume an additive white Gaussian noise (AWGN) channel for each user in the presence of the interference due to the non-orthogonality between the two users' signals. Therefore, successive-interference cancellation (SIC) is exploited to remove the undesired signal of the other user. The outage probability and the biterror rate performance are presented over different signal-to-interference-plus-noise ratio (SINR). Furthermore, Monte-Carlo simulations via Matlab are utilized to verify the results obtained by FPGA, which show exact-close match.
\end{abstract}

Copyright (C) 2020 Insitute of Advanced Engineeering and Science. All rights reserved.

Mohamad A. Ahmed,

College of Electronics Engineering, Ninevah University,

P.O. 41002, Mosul, Iraq.

Email: mohamad.alhabbar@uoninevah.edu.iq

\section{INTRODUCTION}

The next generation of wireless communication $(5 \mathrm{G})$, which will be launched beyond 2020 , will witness high demand on spectrum efficiency and capacity [1,2]. The data traffic of mobile cellular communication systems are expected to increase exponentially, at least thousand time more than the required volume of the last decade [3, 4]. In the previous generations of mobile communication, i.e. $3.9 \mathrm{G}$ and $4 \mathrm{G}$, the technique of orthogonal multiple access (OMA) has been wildly exploited to achieve suitable throughput for single-user performance [5]. Non-orthogonal multiple access (NOMA) is considered one of the most promising multiple access (MA) schemes for download in the 5G [6]. This MA scheme utilizes the power allocation technique in the power domain instead of the time and frequency multiple access, in which the symbols of several users are scaled on the base-station (BS) according to their channels conditions, i.e. the attenuation factor caused due to path loss of each user's channel, then all the scaled symbols are added together and transmitted as one symbol called NOMA symbol [7]. In other words, a user of far distance from the BS will be given more power than a user with near distance. At the far-user (FU), the receiver will deal with near user (NU) signal on the NOMA symbol as a noise, while at the NU, successive interference cancellation (SIC) is required to remove the FU power since the letter is bigger than the intended NU power [8,9]. Furthermore, NOMA with SIC can offer significant user-fairness and better connectivity comparing to the conventional OMA [10].

In the context, NOMA technique is proposed for different applications and mechanism in communications. For instant, NOMA is suggested to be a part of the cooperative communications via relay with the aid of buffering technique [11], and for relays selection to obtain optimum connectivity [12]. Additionally, NOMA 
for two ways half-duplex relaying network is modeled and analyzed in [13] with the aid of decode-and-forward relay. Moreover, NOMA is proposed with massive multiple-input multiple-output (MIMO) relaying, which compared with conventional MIMO-OMA showing significant throughput improvement [14].

In the literature, NOMA based on dynamic scheme is proposed in [15] for direct communications with the users near to a BS with an assistance of relay for far users in the edge of cells. In [16], NOMA based cooperative network is exploited to serve primary and secondary users by a BS, in which spatial diversity is utilized by the BS and among the secondary user to cope the impairments of fading in the channel. The authors in [17] propose NOMA to secure transmissions of two transceivers with their relative destinations over amplify-and-forward cooperative relaying technique. In the other hand, cooperative NOMA via relay is used for maximizing the power efficiency of transmission as suggested in [18], in which a harvested energy of the signals is exploited for sending the information to the intended destination. Moreover, the bit-error rate (BER) in exact-close form is derived in [19] for NOMA over Rayleigh fading channels, considering imperfect SIC in the uplink and downlink.

Field-programmable gate array (FPGA) is an attractive and promising technology which is exploited in this paper to implement the NOMA system in the real-time. This is due to the fact that FPGAs offer more flexibility for the designer for modification of the designed systems with highest available throughput [20]. Moreover, FPGAs is widely used practically in different applications as military radios and the cellular networks infrastructure. In the context, to reduce the latency and complexity, real-time FPGAs is used to implement MIMO long with orthogonal frequency devision multiplexing (OFDM) in [21], in which iterative receiver is suggested. In [22], real-time FPGAs is utilized to design massive multi-user MIMO-OFDM with approximate minimum-mean-square error algorithm for detection. Modern and efficient approach is proposed in [23] to evaluate performance and the consumed power for wireless communication based-FPGAs.

Furthermore, a transceiver based on Spatio-Temporal array-receiver technique for most NOMA radio types is proposed in [24]. This approach offers low complex adaptation and configuration. This mechanism is proposed to tackle the feasibility of hardware and for concept proofing of time-delay estimator based on this technique running over real-time FPGA and MiniBee software defined radio platforms.

Additionally, NOMA is merged with massive-MIMO and millimeter wave (mm-Wave) in [25] to enhance the capacity of the next generation of mobile networks. The analysis of capacity is derived in this research paper, in which the mm-Wave is modeled by using the angle of arriving along with the proposed version of the uniform-random single path model. moreover, the performance is divided into two regions depending on high and low SNRs.

In [26], NOMA system in the download phase along with codeword level SIC are implemented practically via employing software defined radio mechanism by utilizing Open-Air-Interface, in which the proposed system follows the long-term evolution technique in the basic specifications for comparison purposes. Non-regenerative relays are employed in [NonR-Relay] to secure communications with massive-MIMO basestation. The proposed system utilizes NOMA at the transmitters and SIC along with minimum-mean-square error equalization at each receiver. The authors derived exact expression for the SINR, which is used then to evaluate the capacity of the proposed system.

In [27], network coded multiple access is investigated as a new architecture for NOMA. This technique employs near power balance mechanism NOMA to allow several users to share the network in the power domain. This approach can be implemented by applying physical layer network coding along with multi-user decoding to enhance the system capacities.

A compromise solution between bandwidth and power consumption is proposed and investigated in [28] for 5G-Xhual reconfigurable networks, in which traditional radio base-stations radio remote unit are used to handle users' generated traffic, the reconfigurable networks is exploited to carry the sum of the above traffic.

The main goal of this research paper is to experimentally investigate NOMA technique using FPGA over AWGN channel, in which NOMA is applied for two users in the downlink phase via FPGA simulation. Furthermore, power allocation mechanism is employed to share the available power between two users with different distances from the BS, i.e. far and near uses. Without the loss of generality, the assumption of considering only two users in this paper, is due to the fact that regardless of the number of users, the remote user always considers other users' signals as just additive noise as defined by NOMA theory. In other hand, any number of users will follow the same procedure in processing its desired signal as the near user, as they apply SIC to remove signals of other users with higher power allocation factors. Moreover, the performance 
is verified by utilizing Monte-Carlo simulation via Matlab showing exact-closed matching. To the best of our knowledge, this is the first time to implement NOMA system by employing FPGA technique via a code of VHDL programming.

\section{SYSTEM MODELING AND IMPLEMENTATION}

In this paper, a wireless communication is considered for a BS with different distances users NU and FU. We assumed that each terminal is equipped with single antenna as shown in Figure 1. In the downlink phase, the BS generates two quadrature phase shift keying (QPSK) signals for each users. QPSK is chosen in this paper since all the symbols in this modulation scheme have the same absolute value in both the real and imagery parts, which means that they have the same transmitted power. This reduces the complexity of design multi-level amplifier at the receiver and makes the signal processing of much easier.

The signals of each user is multiplied by a factor according to its distance from the BS, i.e. $\beta_{N}$ and $\beta_{F}$ for the near and far users, respectively, where $\beta_{F}>\beta_{N}$. This is due to the assumption that the FU needs to be applied with more power than the NU in order to satisfy users fairness. Moreover, the relationship between the two scaling factors can be expressed as

$$
\beta_{F}+\beta_{N}=1
$$

The received signal at the $K^{\text {th }}$ node can be expressed as

$$
\mathbf{r}_{K}=\mathbf{h}_{K}\left(\sqrt{\beta_{F} P} \mathbf{s}_{F}+\sqrt{\beta_{N} P} \mathbf{s}_{N}\right)+\mathbf{n}_{K}
$$

where $P$ is the total power allocated for the two users, $\mathbf{s}_{K}$ with $K \in(F, N)$ represents a QPSK signal which can take four possible complex values defined as $\mathbf{s}_{K}= \pm 1 \pm j 1$ resulting from mapping two bits. Additionally, $\mathbf{h}_{K}$ denotes a non-selective Rayleigh flat fading channel with zero mean and unity variance, i.e. $\mathbf{h}_{K} \sim \mathcal{C N}(0,1)$, in which the power normalization of the channel between the BS and near user, $\left\|h_{N}\right\|^{2}$, is greater than the normalized power of channel between the BS and the remote user, $\left\|h_{F}\right\|^{2}$, i.e. $\left\|h_{N}\right\|^{2}>\left\|h_{F}\right\|^{2}$. This is due to the attenuation accompanied to wireless propagation of the signals.

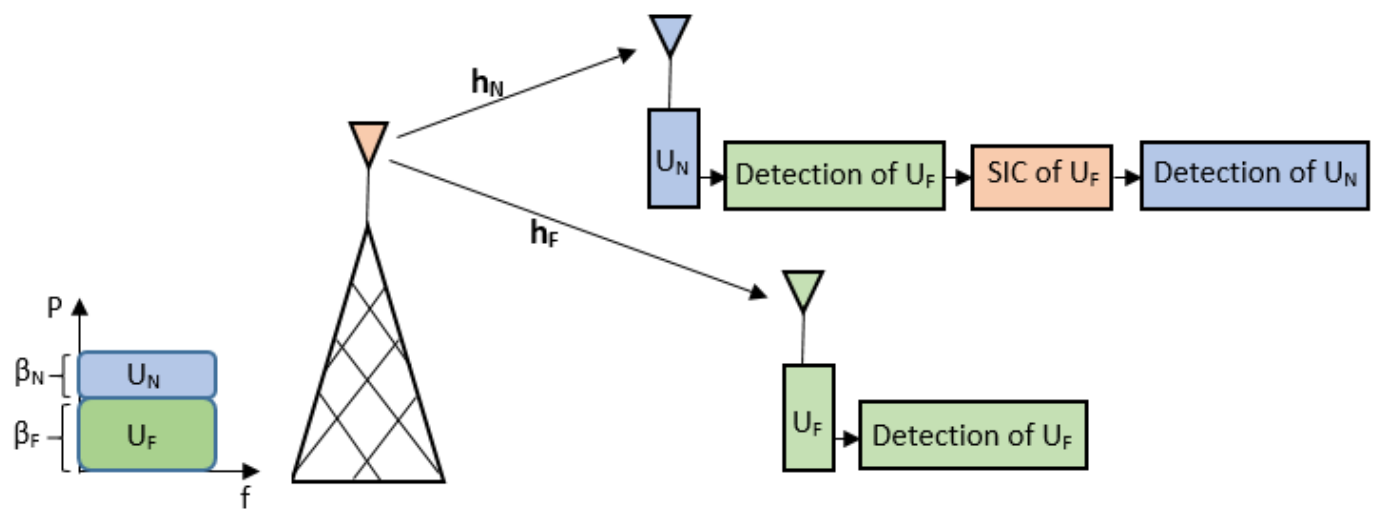

Figure 1. NOMA in the downlink phase for two users, $\left\|h_{N}\right\|^{2}>\left\|h_{F}\right\|^{2}$

Furthermore, $\mathbf{n} \sim \mathcal{C N}\left(0, \sigma_{n}^{2}\right)$ represents a complex distribution of the additive white Gaussian noise (AWGN) with zero mean and a variance of $\sigma_{n}^{2}$ which is added at each node independently. Furthermore, the signal-to-interference-plus-noise ratio (SINR) at the $K^{\text {th }}$ user, which is denoted as $\gamma_{K}$ can be expressed by

$$
\gamma_{K}=\frac{\beta_{K} P\left\|\mathbf{h}_{K} \mathbf{s}_{K}\right\|^{2}}{\beta_{L} P\left\|\mathbf{h}_{K} \mathbf{s}_{L}\right\|^{2}+\left\|\mathbf{n}_{K}\right\|^{2}} \quad L \in\{F, N\} \text { and } \mathrm{L} \neq \mathrm{K}
$$

which can be simplified as

$$
\gamma_{K}=\frac{\Omega_{K}\left\|\mathbf{h}_{K}\right\|^{2}}{\Omega_{L}\left\|\mathbf{h}_{K}\right\|^{2}+1}
$$


where $\Omega_{K}=\frac{\beta_{K} P}{\sigma_{n}^{2}}$ and $\Omega_{L}=\frac{\beta_{L} P}{\sigma_{n}^{2}}$ represent the signal-to-noise ratio (SNR) and the interference-to-noise ratio (INR) at the user of interested. Therefore, the Shannon capacity for this system can be evaluated for a particular $K^{\text {th }}$ user as

$$
C_{K}=\frac{1}{2} \log _{2}\left(1+\gamma_{K}\right)
$$

\section{NOMA IMPLEMENTATION VIA FPGA}

The implementation is achieved by utilizing Spartan 3e, which has a slice number Xc3s500e. The starter kit board of Spartan 3e family of FPGA has an embedded convenient development board, for various signal processing applications. This board is designed to meet the high demand on low cost and high volume electronics applications [29]. Figure 2 shows the implemented system, in which the transmitter and the two receivers will be discussed in more details in this paper. Furthermore, Figure 7 and Figure 8 represent the output at each stages of the designed system with FU and NU detections, respectively. Firstly, QPSK symbols are generated according to the transmitted bits of the NU and FU, respectively. These QPSK symbols for each users are then scaled by $\sqrt{\beta_{N}}$ and $\sqrt{\beta_{F}}$. The two scaled symbols for the two users are then added together to create NOMA symbols, which transmitted over two independent AWGN channels. The implementation of AWGN channel is discussed in the next subsection in more details. Moreover, in our FPGA design, $\beta_{N}$ and $\beta_{F}$ can take any values depending on the change of the distances of NU and FU from the base-station by applying (1). However, optimization techniques can be employed to find the best values which is out of the scope of this paper. Figure 2 represents the entire designed NOMA transmitter along with the FU and NU receivers by using FPGA. All the defined inputs/outputs (I/O)s with the block diagram for each stage of this process are illustrated clearly. Moreover, it is noteworthy that two frequencies are used in our design which are $50 \mathrm{MHz}$ and $12.5 \mathrm{MHz}$ for the purpose of synchronization. The two frequencies are generated by employing frequency divider technique of the clock generator.

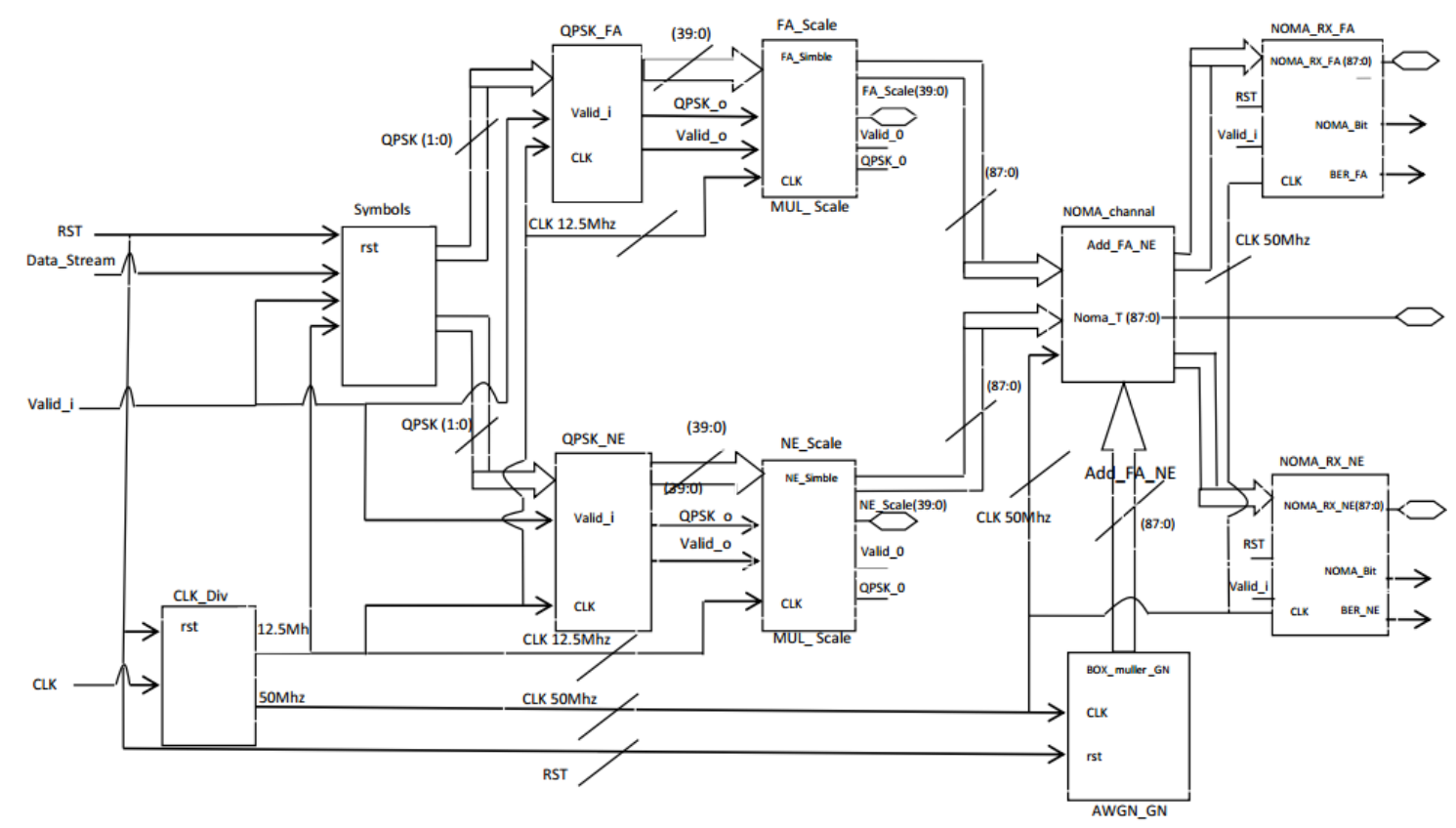

Figure 2. The entire designed of NOMA transmitter along with the FU and NU receivers via FPGA, the signals names, abbreviations and functions are illustrated in Table 1 
Table 1. Signals abbreviations, names with their functions as used in Figure 2

\begin{tabular}{|c|c|c|}
\hline Signal Abbreviation & Signal Name & Function of the signal \\
\hline RST & Reset & Reset system \\
\hline Data-Stream & Data Stream & Serial data stream Input \\
\hline Valid $_{i}$ & Valid Input & When this signal is " 1 " the data stream input will be read \\
\hline CLK & Clock & $\begin{array}{c}\text { but when this signal is " } 0 \text { " the data stream input } \\
\text { will not be read, it just ignored. } \\
\text { System clock }(50 \mathrm{MHz})\end{array}$ \\
\hline CLK-Div & Clock divider & System clock $(50 \mathrm{MHz}, 12.5 \mathrm{MHz})$ \\
\hline QPSK(1:0) & Quadrature Phase & Two bits of QPSK signal for output symbol block \\
\hline & Shift Keying bits & \\
\hline QPSK-o & QPSK output & QPSK output clock for synchronization \\
\hline Valid-o & Valid Output & Valid out to enable receiving data input stream \\
\hline QPSK-FA & QPSK for Far user & Mapping of two bits to a QPSK Symbol for Far user \\
\hline QPSK-NR & QPSK for Near user & Mapping of two bits to a QPSK Symbol for Near user \\
\hline FA-Scale & Far Scale & Far user symbol Scaling using power \\
\hline NE-Scale & Near Scale & $\begin{array}{l}\text { allocation technique } \\
\text { Near user symbol Scaling using power }\end{array}$ \\
\hline FA-Scale $(39: 0)$ & Far Scale for 40 bits & $\begin{array}{l}\text { allocation technique } \\
\text { Far Scale Indication }\end{array}$ \\
\hline NE-Scale (39:0) & Near Scale for 40 bits & Near Scale Indication \\
\hline NOMA-Channel & NOMA channel & Add Far and Near symbols along with \\
\hline BOX-Muller-GN & Box muller Generation & $\begin{array}{l}\text { adding the AWGN generated by Box Muller method } \\
\text { Block diagram responsible for }\end{array}$ \\
\hline NOMA-RX-FA & Received Far & $\begin{array}{l}\text { AWGN symbol generation } \\
\text { The received NOMA signal for the Far user }\end{array}$ \\
\hline NOMA-RX-NE & $\begin{array}{l}\text { user NOMA } \\
\text { Received Near } \\
\text { user NOMA }\end{array}$ & The received NOMA signal for the Near user \\
\hline NOMA-Bit & NOMA bit & $\begin{array}{l}\text { The bits after symbol detection of the Far or Near user } \\
\text { corresponding on the user's receiver. }\end{array}$ \\
\hline BER-FA & Bit error rate Far & Bit error rate for Far signal \\
\hline BER-NE & Bit error rate Near & Bit error rate for Near signal \\
\hline
\end{tabular}

Figure 3 shows the percentage values of the consumed number of slices, flip-flops, 4-input lookup tables (LUT)s, bounded input/output blocks (IOB)s and GCLKs in the utilized Xc3s500e Spartan-3e kit. In this figure, available and the utilization number of each of the above parameters are illustrated after applying optimization to the designed system. It is clear that all utilization percentages are lower than the maximum available capacity of the kit, except the number of the bounded IOBs which is just below the maximu number. This issue can be tackled by using the upgraded version Xc3s500e Spartan-3e kit with higher gates.

\begin{tabular}{|l|r|r|r|r|}
\hline \multicolumn{4}{|c|}{ Device Utilization Summary (estimated values) } & Utilization \\
\hline Logic Utilization & Used & Available & 4656 & $15 \%$ \\
\hline Number of Slices & 705 & 9312 & $0 \%$ \\
\hline Number of Slice Flip Flops & 1290 & 9312 & $13 \%$ \\
\hline Number of 4 input LUTs & 229 & 232 & $98 \%$ \\
\hline Number of bonded IOBs & 2 & 24 & $8 \%$ \\
\hline Number of GCLKs & & & \\
\hline
\end{tabular}

Figure 3. The percentage values of the consumed number of components in Xc3s500e Spartan-3e FPGA kit 
In order to add AWGN to the created NOMA symbols in FPGA, we follow the Box-Muller technique which is widely exploited in different software for this purpose as in [30, 31]. In brief, two random variables $x_{1}$ and $x_{2}$, which have uniform distribution between 0 and 1 , are employed to create random Gaussian noise sample, with zero mean and standard divination of $\sigma^{2}$, afterwards, two functions $f\left(x_{1}\right)$ and $g\left(x_{2}\right)$ are derived from the two variables as

$$
\begin{aligned}
f\left(x_{1}\right) & =\sqrt{-\ln x_{1}}, \\
g\left(x_{2}\right) & =\sqrt{2} \cos \left(2 \pi x_{2}\right), \\
n & =f\left(x_{1}\right) g\left(x_{2}\right),
\end{aligned}
$$

where $n$ represents the AWGN symbol created from the two functions $f\left(x_{1}\right)$ and $g\left(x_{2}\right)$ according to the two random variables defined above [30, 31], respectively.

In order to satisfy small variation of $\mathbf{x}_{1}$, non-uniform quantization can be used, as proposed in [32], by portioning the segment $[0,1]$ recursively into $M=16$ subsegment having the same length. Each of these segments are then divided again into subsegments. This process is implemented $L$ times using 1024 bytes ROM to store $f\left(\mathbf{x}_{1}\right)$ which has the quantized values $F_{r}(s)$ at each level of partitioning. This can be expressed as

$$
F_{r}(s)=R\left\lfloor 2^{m} f\left(\frac{s+\zeta}{16^{r}}\right)\right\rfloor \times\left(2^{-m}\right),
$$

where $r$ has a range between 1 to $L$, while $s$ varies between 1 to $M-1$. Moreover, $\zeta$ can be defined as a real number within the range $[0,1]$ to indicate the position of each sample. It is noteworthy that if $F_{r}(s)$ is used to quantize $(\kappa+m)$ bits, then $\kappa$ represents the integer value to obtain $\sigma=2^{\kappa}$, while $m$ is to represents the fractional part. Furthermore, $\lfloor R\rfloor$ is the maximum integer below $x$. Additionally, for $s=0$ in $(7) F_{r}(0)=0$. Now, the variable $x_{1}$ can be obtained by using $L$ random generator of 4 bits, i.e. $r g_{r}$ with $r=1$ to $L$. The quantization function of the second variable $g\left(x_{2}\right)$ can be simplified by exploiting the symmetrical property of the Cosine function. The quantization function can be expressed as

$$
G\left(s^{\prime}\right)=R\left\lfloor 2^{m^{\prime}} \sqrt{2} \cos \left(\frac{\pi\left(s^{\prime}+\zeta^{\prime}\right)}{2}\right)\right\rfloor \times\left(2^{-m^{\prime}}\right),
$$

where $m^{\prime}$ and $\zeta^{\prime}$ are as defined already in (7), $s^{\prime}$ is a random variable represented by 8 bits. Similarly, $G\left(s^{\prime}\right)$ is used to quantize $\left(1+m^{\prime}\right)$ bits, where 1 and $m^{\prime}$ are to represent the integer and fraction parts, receptively. After obtaining $F_{r}(s)$ and $G\left(s^{\prime}\right)$, the random variable of the noise can be created using half Boxed-Muller with $b$ bits as

$$
n^{+}=\left\lfloor\frac{F_{r}(s) G\left(s^{\prime}\right)}{2^{\left(m+m^{\prime}-b\right)}}\right\rfloor \times\left(2^{b}\right),
$$

which can be used to create the complete Boxed-Muller random variable of the noise by applying a binary random variable sign as

$$
n=(1-2 \times \operatorname{sign}) n^{+}
$$

In our design, we exploit Box-Muller method as illustrated in Figure 4 to generate random independent AWGN for each user with different initial seed. In this figure, random stream of bits are generated to feed into two 8 bits linear feedback shift registers (LFSR). The designed LFSR is shown Figure 5 which follows the polynomial $\left(x^{8}+x^{5}+x^{3}+x+1\right)$. Moreover, a logical delay, denoted by $D$, is applied to one of the two branches to ensure no correlation with the other branch. The 16 bits LUT is used to evaluate the equation $V_{\text {out }}=A+A \cos (w t)$ where $A=2.5$ volt here in order to achieve $V_{\text {out }}=5$ volt when $\cos (w t)=1$, and $V_{\text {out }}=0$ volt when $\cos (w t)=-1$. Furthermore, the exploited frequency is $12.5 \mathrm{MHz}$ and for 10 bits serial peripheral interface (SPI), which is used to increase the resolution and reduce the processing time for the analogue to digital converter (ADC) instead of using the conventional serial to parallel technique. The output of each LUT is multiplied by a 16-bits sign, then they added together and pass the output to a register with a length of 11 bytes, i.e. $(0-87)$ bits. This output represents the generated AWGN complex symbols with 
zero mean and variance equal to $\sigma_{n}^{2}$ as shown in Figure 6 for some symbols with $\mathrm{SNR}=15 \mathrm{~dB}$. It is worth to note that the same procedure is applied at the other user with different seed to ensure independent AWGN.

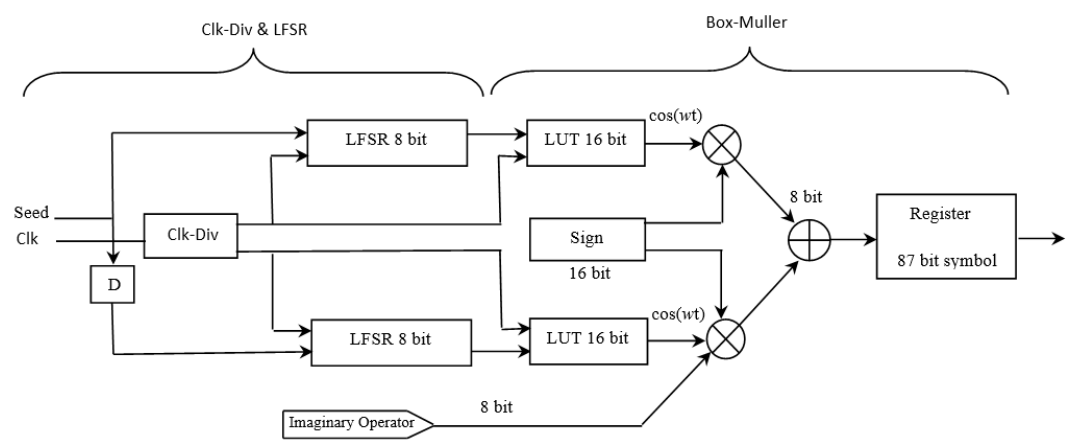

Figure 4. Box-Muller method used to generate AWGN in each user's receiver

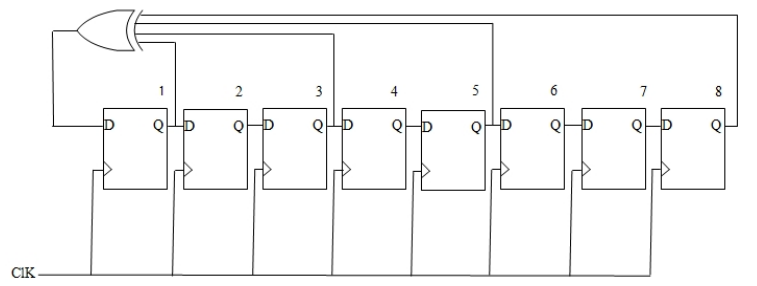

Figure 5. The schematic diagram of the 8 bits LFSR with a polynomial $\left(x^{8}+x^{5}+x^{3}+x+1\right)$ which employed in Box-Muller approach to generate AWGN
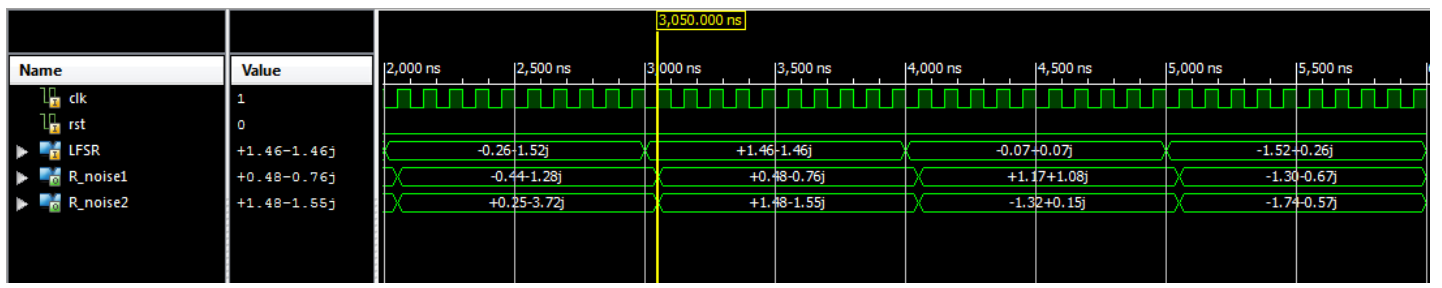

Figure 6. Samples of the generated AWGN by using Box-Muller technique

\section{DETECTION OF NOMA}

In the detection of NOMA signal, which contains a non-orthogonal combination of the multiple users, different techniques are exploited for the near and far users depending on the level of interference caused by the other user. At the FU terminal, in which the far signal is strong while the near signal is weak, simple and ordinary technique can be used for detection by considering the weak NU signal as a noise. In contrast, at the NU terminal, advanced mechanism should be used to detect the NU weak signal, due to the existence of the strong FU signal. This can be implemented by applying SIC. In brief, SIC is simply utilized by detecting the FU signal at the NU terminal and subtract it from the overall NOMA signal to obtain the NU signal.

\section{SIMULATIONS RESULTS AND DISCUSSION}

In this section, the simulation results are obtained for the designed NOMA system in FPGA for a BS communicating with two users, NU and FU. We verify the results by utilizing Monte-Carlo simulation via Matlab for the same system over AWGN channel and with different SNR and power allocation scales. We assume that the attenuation between the BS and NU is $0 \mathrm{~dB}$, while it is $-3 \mathrm{~dB}$ between the BS and FU. Without the loss of generality, downlink phase is considered for the two users in this paper, i.e. for the far and 
near distances from the base-station, in which the FU is applied with larger portion of the available power of NOMA signal than the NU. This is due to the fact that the signal losses part of its power as long as the distance is increased, this what is called attenuation phenomena.

Figure 7 and Figure 8 show the FPGA signaling diagram for the simulated NOMA BS transmitter with FU and NU receivers, respectively, in the downlink phase. First of all, a series of bits are generated to represent the data stream for each user. Moreover, the power allocation factors for the NU, and FU, which are denoted as $n e-$ scale $=\sqrt{\beta_{N}}=\sqrt{0.4}=0.63$ and $f a-$ scale $=\sqrt{\beta_{F}}=\sqrt{0.6}=0.77$, respectively, are chosen. The bits are converted to QPSK symbols. i.e. every two bits are converted to a symbol to take one of the four possible symbols $(\mp 1 \mp j 1$ ), and then multiplied by the assigned scale depending on the channel condition of each user. The scaled symbols of NU and FU are added together to create the NOMA symbols. The latter are sent towards each user over AWGN channel with different attenuation factors. Additionally, in this figure, the arrived NOMA symbols at the FU terminal are illustrated and the detected symbols after comparing each symbol with the nearest expected location on the original QPSK constellation. Finally, the received symbols at FU receiver are compared with the transmitted symbols, and any difference leads to create an error signal, denoted by ber which are summed and divided by the total number of bits to obtain the symbols error rate (SER) at each SNR.

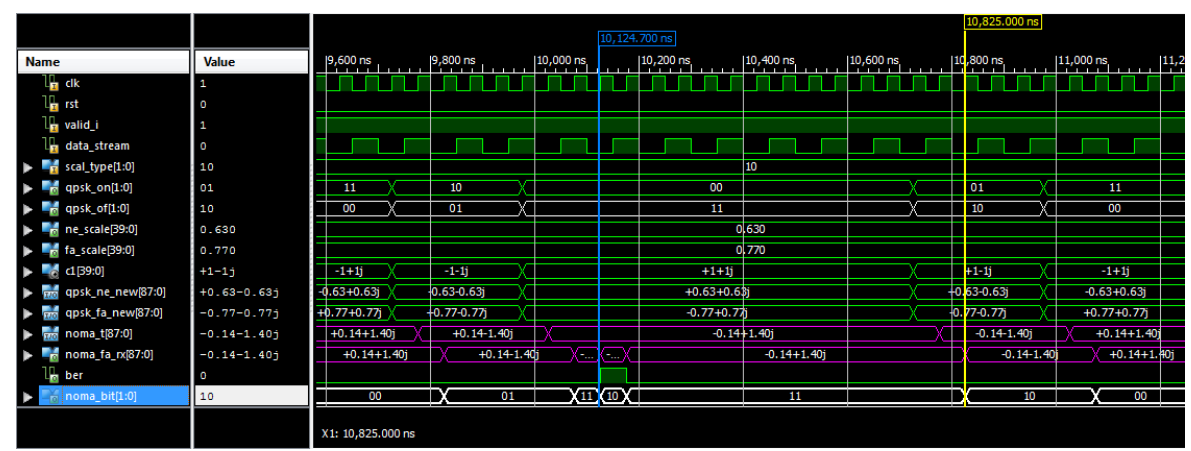

Figure 7. Base-station NOMA transmitter timing as implemented via FPGA and the FU detection

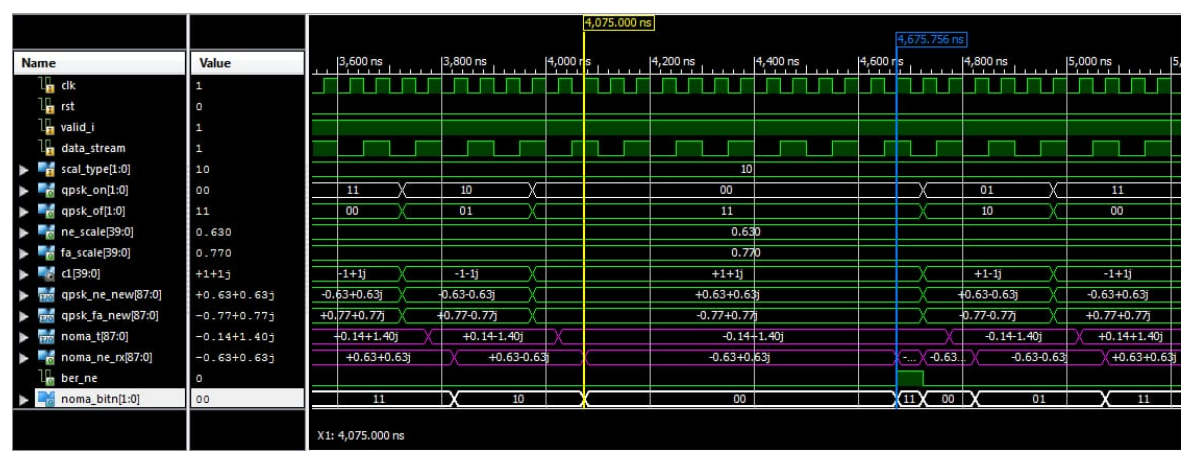

Figure 8. Base-station NOMA transmitter timing as implemented via FPGA and the NU detection by applying SIC

In Figure 9, the effect of the AWGN on a symbol is clearly illustrated when $\mathrm{SNR}=15 \mathrm{~dB}$. This figure shows the error in detection a symbol transmitted from the BS as two successive logic 1, i.e. 11, while in the FU receiver is detected it wrongly as 10 because of the noise. The designed system detect this error and a signal ber is generated for the purpose counting the number of errors as mentioned previously. Figure 10 shows the output of FPGA simulation similar to Figure 9, but for the NU. The detection at this user begins with detection of the FU signal at the near terminal as explained previously, followed by subtracting the FU from the entire NOMA signal received obtain NU symbols. The latter are mapped again to their nearest positions in the QPSK constellation. The symbol errors which are taken place in this process between the transmitted and received 
symbols are detected by creating the error signal, ber, which is summed and divided by the total number of bits to evaluate the SER. It is worth to mention that the SER is divided by $\log _{2}(4)$ for QPSK scheme to obtain bit error rate $(\mathrm{BER})$ for each user.

The effect of the AWGN of SNR= $15 \mathrm{~dB}$ on a symbol is clearly illustrated in Figure 10. In this figure the error in detection a symbol transmitted as 00 by the BS is shown along with its wrong detection as 11 because of the noise. Again, the designed system detect this error by creating a signal ber to be used to calculate the system BER vs. SNR.

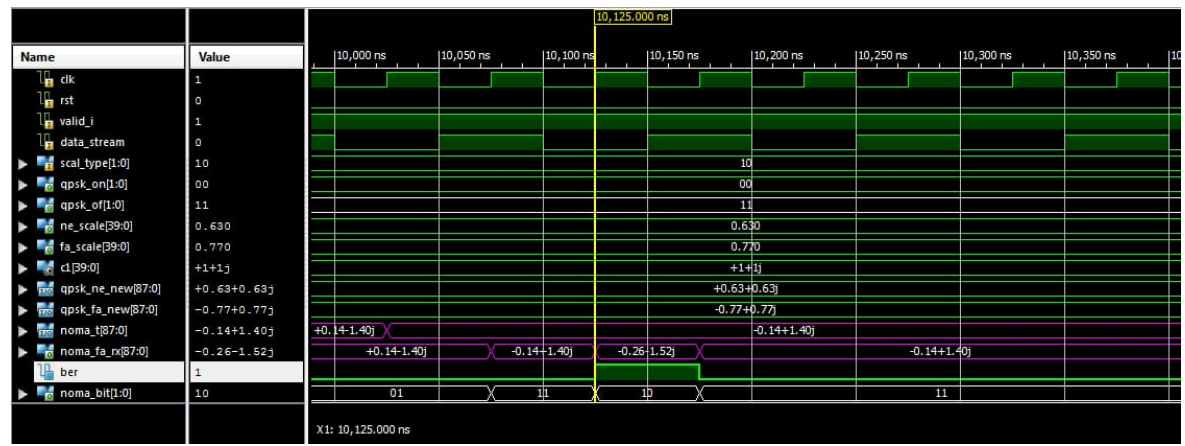

Figure 9. The detection of error symbol for the FU receiver

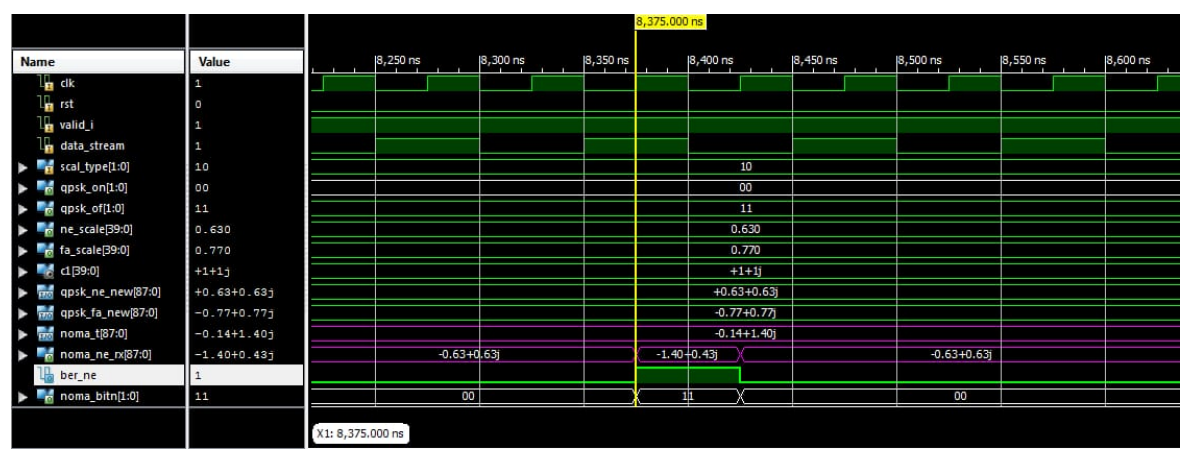

Figure 10. The detection of error symbol for the NU receiver

In Figure 11, Monte-Carlo simulations via Matlab is used to obtain the BER over different SNR to validate the results obtained via FPGA. The used power allocation factors of $\beta_{F}=0.6$ with $\beta_{N}=0.4$ and $\beta_{F}=0.8$ with $\beta_{N}=0.2$ are chosen for the FU and NU, respectively. The detection of the FU signal is implemented by calculating the minimum Euclidean distances between the received symbols and the original symbols in the QPSK constellation. Moreover, the NU signal is assumed as a noise at the FU terminal. In contrast, the SIC is applied at the NU receiver to remove the strong FU signal and detect the weak NU signal. The Euclidean distance of each detected symbols with the original QPSK constellation points are evaluated, where the minimum distance refers to the most likely right position. In this simulation, It is found that the power allocation factors of $\beta_{N}=0.4$ and $\beta_{F}=0.6$ lead to obtain the same BER-SNR performance especially at high SNRs, and increasing $\beta_{F}=0.6$ with decreasing $\beta_{N}=0.4$ lead to obtain better performance in the Far terminal. This is in order to satisfy some sort of user-fairness in this performance metric by applying power allocation technique to compensate for the channel weakness. On other words, for different normalized power of the near and far channels as defined in this paper $\left\|h_{N}\right\|^{2}>\left\|h_{F}\right\|^{2}$, where $\left\|h_{N}\right\|^{2}=0 \mathrm{~dB}$ and $\left\|h_{F}\right\|^{2}=-3$ $\mathrm{dB}$, we found that when $\beta_{N}=0.4$ and $\beta_{F}=0.6$, the two users have the same BER-SNR performance. On the other hand, when $\beta_{F}$ is increased and $\beta_{N}$ is decreased, satisfying $\beta_{F}+\beta_{N}=1$, this will lead to $U_{F}$ outperforming $U_{N}$. Moreover, these results show exact-closed matching with the results obtained from FPGA simulations after using the same parameters. 
Figure 12 shows the performance of the system using the outage probability against the SNR for a threshold BER of $10^{-4}$. Three sceneries have been taken into account, which are $\beta_{F}=0.6$ with $\beta_{N}=0.4$, $\beta_{F}=0.7$ with $\beta_{N}=0.3$ and $\beta_{F}=0.8$ with $\beta_{N}=0.2$. The channels conditions are assumed as $\left\|h_{N}\right\|^{2}=0$ $\mathrm{dB}$ and $\left\|h_{F}\right\|^{2}=-3 \mathrm{~dB}$. It can be noticed that the performance of the far user outperforms the near one when $\beta_{F}=0.8$ with $\beta_{N}=0.2$. Some sort of convergence in the outage probability can be seen when $\beta_{F}=0.7$ with $\beta_{N}=0.3$ and $\beta_{F}=0.6$ with $\beta_{N}=0.4$, for SNR below $23 \mathrm{~dB}$ and $28 \mathrm{~dB}$, respectively. While after these two values of SNRs the far user performs better.

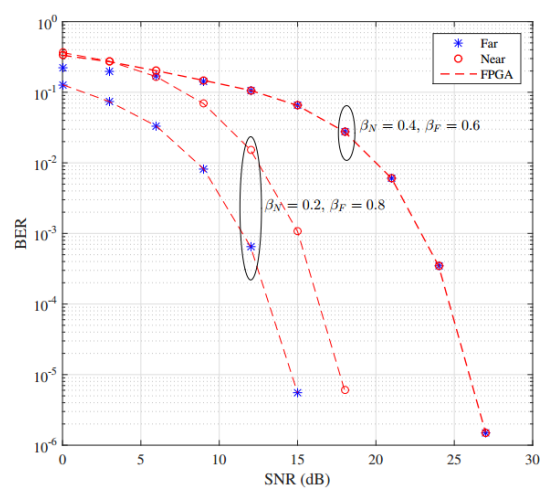

Figure 11. BER vs. SNR for two-user NOMA-QPSK in the downlink phase with $\beta_{F}=0.6$ with $\beta_{N}=0.4$ and $\beta_{F}=0.8$ with $\beta_{N}=0.2$, for $\left\|h_{N}\right\|^{2}=0 \mathrm{~dB}$ and $\left\|h_{F}\right\|^{2}=-3 \mathrm{~dB}$

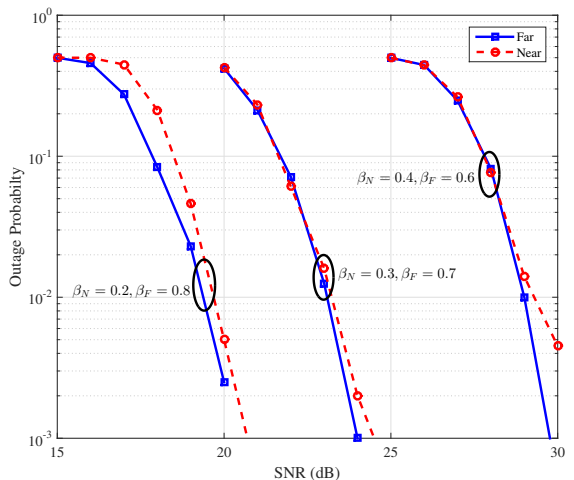

Figure 12. The outage probability vs. SNR for two-user NOMA-QPSK in the downlink phase with $\beta_{F}=0.6$ with $\beta_{N}=0.4$, $\beta_{F}=0.7$ with $\beta_{N}=0.3$ and $\beta_{F}=0.8$ with $\beta_{N}=0.2$, for $\left\|h_{N}\right\|^{2}=0 \mathrm{~dB}$ and

$$
\left\|h_{F}\right\|^{2}=-3 \mathrm{~dB} \text { and for a BER }
$$
threshold of $10^{-4}$

Moreover, the constellation of the received NOMA signal at both users is shown in Figure 13. This constellation is already generated in the base-station by adding two QPSK symbols with different power allocation factors, i.e. $\beta_{N}$ and $\beta_{F}$ for the near and far users, respectively. Furthermore, Figure 14 shows the symbols after applying SIC at the NU receiver. It is clear that the 16-NOMA symbols in Figure 13 have become 4 symbols in Figure 14, referring to the QPSk symbols, after applying the SIC, in which the symbols of the remote user with higher power allocation factor is detected and subtracted from the entire 16-NOMA symbols at the near terminal to obtain the QPSK symbols of the near user only.

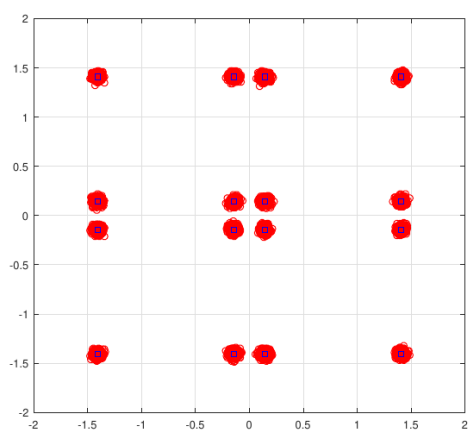

Figure 13. Downlink NOMA constellation created from adding two QPSK symbols with different power allocation factors i.e. $\beta_{F}=0.6$ and $\beta_{N}=0.4$ at the NU front-end before applying SIC, the blue symbols refer to the original 16-NOMA constellation at the BS before adding the noise

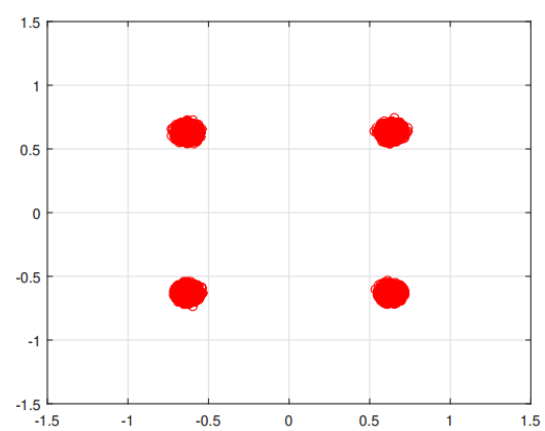

Figure 14. Near user QPSK constellation at the NU receiver after applying SIC to the NOMA signal with $\beta_{F}=0.6$ and $\beta_{N}=0.4$ 
The throughputs of the two users are shown in Figure 15 for the downlink phase by using (5) over a range of SNR from $(0-30) \mathrm{dB}$. It can be noted that applying SIC to the NU improve the overall system capacity significantly.

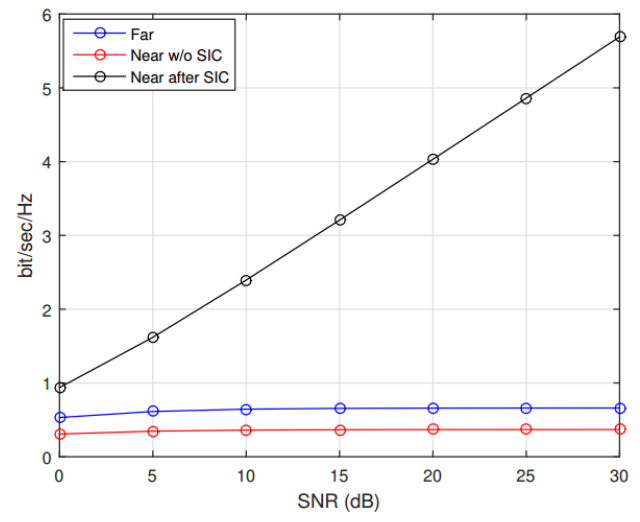

Figure 15. Capacity for the two user and after using SIC at the NU, $\beta_{F}=0.6$ and $\beta_{N}=0.4$

\section{CONCLUSIONS}

In this paper, NOMA technique is applied to secure a user-fairness and connectivity for two users with the BS over AWGN. FPGA is used to design the system in the downlink phase and to evaluate performance of the designed system. The obtained performance has been compared with Monte-Carlo simulations results. An exact-closed matching is observed for the BER-SNR performance metric for different SNR and power allocation scales for the two user. Moreover, SIC has been employed at the receiver of the near user to remove the strong signal of the far user. In contrast, the far user's receiver considers the weak signal of the near user as a noise during its detection of the far user signal. Additionally, AWGN channels are assumed between the two users and the BS over different SNR. The throughput of the two users are evaluated which shows the outperforming of the near user despite lower power allocation factor, since it has stronger channel. The consumed percentage of the number of gates and shift register are tabulated showing estimated values summery of the device utilization.

\section{REFERENCES}

[1] V. K. Trivedi, K. Ramadan, P. Kumar, M. I. Dessouky, and F. E. A. El-Samie, "Enhanced ofdm-noma for next generation wireless communication: A study of papr reduction and sensitivity to cfo and estimation errors," AEU-International Journal of Electronics and Communications, 2019.

[2] B. Selim, S. Muhaidat, P. C. Sofotasios, B. S. Sharif, T. Stouraitis, G. K. Karagiannidis, and N. Al-Dhahir, "Performance analysis of non-orthogonal multiple access under i/q imbalance," IEEE Access, vol. 6, pp. 18 453-18 468, 2018.

[3] J.-B. Kim and I.-H. Lee, "Non-orthogonal multiple access in coordinated direct and relay transmission," IEEE Communications Letters, vol. 19, no. 11, pp. 2037-2040, 2015.

[4] G. Niharika, "Performance comparison of modulation schemes for downlink noma," in 2018 2nd International Conference on Inventive Systems and Control (ICISC). IEEE, 2018, pp. 1431-1433.

[5] H. Tabassum, M. S. Ali, E. Hossain, M. J. Hossain, and D. I. Kim, "Uplink vs. downlink noma in cellular networks: Challenges and research directions," in 2017 IEEE 85th Vehicular Technology Conference (VTC Spring). IEEE, 2017, pp. 1-7.

[6] M. M. El-Sayed, A. S. Ibrahim, and M. M. Khairy, "Power allocation strategies for non-orthogonal multiple access," in Selected Topics in Mobile \& Wireless Networking (MoWNeT), 2016 International Conference on. IEEE, 2016, pp. 1-6.

[7] S. R. Islam, N. Avazov, O. A. Dobre, and K.-S. Kwak, "Power-domain non-orthogonal multiple access (noma) in 5g systems: Potentials and challenges," IEEE Communications Surveys \& Tutorials, vol. 19, no. 2, pp. 721-742, 2017. 
[8] Y. Chen, L. Wang, Y. Ai, B. Jiao, and L. Hanzo, "Performance analysis of noma-sm in vehicle-to-vehicle massive mimo channels," IEEE Journal on Selected Areas in Communications, vol. 35, no. 12, pp. 26532666, 2017.

[9] Y. Chen, A. Bayesteh, Y. Wu, B. Ren, S. Kang, S. Sun, Q. Xiong, C. Qian, B. Yu, Z. Ding et al., "Toward the standardization of non-orthogonal multiple access for next generation wireless networks," IEEE Communications Magazine, vol. 56, no. 3, pp. 19-27, 2018.

[10] K. Higuchi and A. Benjebbour, "Non-orthogonal multiple access (noma) with successive interference cancellation for future radio access," IEICE Transactions on Communications, vol. 98, no. 3, pp. 403414, 2015.

[11] Z. Liang, X. Chen, and J. Huang, "Non-orthogonal multiple access with buffer-aided cooperative relaying," in 2016 2nd IEEE International Conference on Computer and Communications (ICCC). IEEE, 2016, pp. 1535-1539.

[12] N. Nomikos, T. Charalambous, D. Vouyioukas, G. K. Karagiannidis, and R. Wichman, "Relay selection for buffer-aided non-orthogonal multiple access networks," in 2017 IEEE Globecom Workshops (GC Wkshps). IEEE, 2017, pp. 1-6.

[13] X. Yue, Y. Liu, S. Kang, A. Nallanathan, and Y. Chen, "Modeling and analysis of two-way relay nonorthogonal multiple access systems," IEEE Transactions on Communications, vol. 66, no. 9, pp. 37843796, 2018.

[14] D. Zhang, Y. Liu, Z. Ding, Z. Zhou, A. Nallanathan, and T. Sato, "Performance analysis of nonregenerative massive-mimo-noma relay systems for 5g," IEEE Transactions on Communications, vol. 65 , no. 11, pp. 4777-4790, 2017.

[15] Y. Xu, G. Wang, L. Zheng, and S. Jia, "Performance of noma-based coordinated direct and relay transmission using dynamic scheme," IET Communications, vol. 12, no. 18, pp. 2231-2242, 2018.

[16] S. Bhattacharjee, T. Acharya, and U. Bhattacharya, "Noma inspired multicasting in cognitive radio networks," IET Communications, vol. 12, no. 15, pp. 1845-1853, 2018.

[17] Y. Li, Y. Li, Y. Chen, Y. Ye, and H. Zhang, "Performance analysis of cooperative noma with a shared af relay," IET Communications, vol. 12, no. 19, pp. 2438-2447, 2018.

[18] Y. Zhang, J. He, S. Guo, and F. Wang, "Energy efficiency maximisation in wireless powered networks with cooperative non-orthogonal multiple access," IET Communications, vol. 12, no. 18, pp. 2374-2383, 2018.

[19] F. Kara and H. Kaya, "Ber performances of downlink and uplink noma in the presence of sic errors over fading channels," IET Communications, vol. 12, no. 15, pp. 1834-1844, 2018.

[20] T. H. Pham, S. A. Fahmy, and I. V. McLoughlin, "An end-to-end multi-standard ofdm transceiver architecture using fpga partial reconfiguration,” IEEE Access, vol. 5, pp. 21 002-21 015, 2017.

[21] L. Boher, R. Rabineau, and M. Helard, "Fpga implementation of an iterative receiver for mimo-ofdm systems," IEEE Journal on Selected Areas in Communications, vol. 26, no. 6, pp. 857-866, 2008.

[22] M. Wu, C. Dick, J. R. Cavallaro, and C. Studer, "High-throughput data detection for massive mu-mimoofdm using coordinate descent," IEEE Transactions on Circuits and Systems I: Regular Papers, vol. 63, no. 12, pp. 2357-2367, 2016.

[23] J. Lorandel, J.-C. Prévotet, and M. Hélard, "Fast power and performance evaluation of fpga-based wireless communication systems," IEEE Access, vol. 4, pp. 2005-2018, 2016.

[24] H. Haggui, F. Bellili, and S. Affes, "Fpga prototyping of a star-based time-delay estimator for $5 \mathrm{~g}$ radio access," in 2017 IEEE 28th Annual International Symposium on Personal, Indoor, and Mobile Radio Communications (PIMRC). IEEE, 2017, pp. 1-5.

[25] D. Zhang, Z. Zhou, C. Xu, Y. Zhang, J. Rodriguez, and T. Sato, "Capacity analysis of noma with mmwave massive mimo systems," IEEE Journal on Selected Areas in Communications, vol. 35, no. 7, pp. 16061618, 2017.

[26] X. Wei, H. Liu, Z. Geng, K. Zheng, R. Xu, Y. Liu, and P. Chen, "Software defined radio implementation of a non-orthogonal multiple access system towards 5g," IEEE Access, vol. 4, pp. 9604-9613, 2016.

[27] H. Pan, L. Lu, and S. C. Liew, "Practical power-balanced non-orthogonal multiple access," IEEE Journal on Selected Areas in Communications, vol. 35, no. 10, pp. 2312-2327, 2017.

[28] V. Eramo, M. Listanti, F. G. Lavacca, P. Iovanna, G. Bottari, and F. Ponzini, "Trade-off between power and bandwidth consumption in a reconfigurable xhaul network architecture," IEEE Access, vol. 4, pp. 9053-9065, 2016. 
[29] S. Popescu, A. Gontean, and G. Budura, "Bpsk system on spartan 3e fpga," in 2012 IEEE 10th International Symposium on Applied Machine Intelligence and Informatics (SAMI). IEEE, 2012, pp. 301-306.

[30] D.-U. Lee, J. D. Villasenor, W. Luk, and P. H. W. Leong, "A hardware gaussian noise generator using the box-muller method and its error analysis," IEEE Transactions on Computers, vol. 55, no. 6, pp. 659-671, 2006.

[31] J.-L. Danger, A. Ghazel, E. Boutillon, and H. Laamari, "Efficient fpga implementation of gaussian noise generator for communication channel emulation," in The 7th IEEE International Conference on Electronics, Circuits and Systems, 2000. ICECS 2000, vol. 1. IEEE, 2000, pp. 366-369.

[32] A. Ghazel, E. Boutillon, J.-L. Danger, G. Gulak, and H. Laamari, "Design and performance analysis of a high speed awgn communication channel emulator," in Communications, Computers and signal Processing, 2001. PACRIM. 2001 IEEE Pacific Rim Conference on, vol. 2. IEEE, 2001, pp. 374-377. 